\title{
Are Brachiocephalic Atherosclerotic Plaques to be considered as Predictors of Myocardial Infarction in Rheumatoid Arthritis Patients?
}

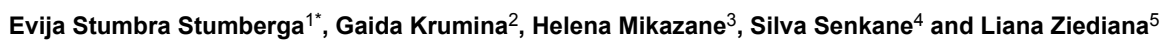

${ }^{1}$ Rheumatology Department, Riga 1st Hospital, Riga, Latvia

${ }^{2}$ Radiology Unit, Riga Eastern Hospital, Riga, Latvia

3 Internal Medicine Department, Riga Stradins University, Riga, Latvia

${ }^{4}$ Statistics Department, Riga Stradins University, Riga, Latvia

${ }^{5}$ Diagnostics Department, Health Center 4, Riga, Latvia

*Corresponding author: Evija Stumbra Stumberga, Rheumatology Department, Gertrudes 69/71, 39, Riga LV 1011, Latvia, Tel: +37126108929; Fax: +37126108929; Email: evijastumbra@inbox.Iv

Received date: July 31, 2017; Accepted date: August 22, 2017; Published date: August 24, 2017

Copyright: (c) 2017 Stumberga ES, et al. This is an open-access article distributed under the terms of the Creative Commons Attribution License, which permits unrestricted use, distribution, and reproduction in any medium, provided the original author and source are credited.

\begin{abstract}
Introduction: Carotid ultrasound based on assessment of intima media thickness (IMT) and presence of plaques has been considered to be a cheap and efficient way to measure clinical atherosclerosis. Both carotid IMT and carotid plaques have been proved to be good predictors of CV (cardiovascular) events of rheumatic patients, offering additional value to the traditional risk scores in the prediction of CVD (cardiovascular disease). The objective of the study was to determine whether atherosclerotic brachiocephalic plaques, traditional CV risk factors as well as RA (rheumatoid arthritis) disease activity have an effect on the risk of myocardial infarction in RA patients with comparable disease duration.
\end{abstract}

Methods: A case control study was performed within the prospective cohort of 92 RA patients, out of them were female $81 \%$, aged $40-84$. In cases there were 20 patients who had developed their first myocardial infarction (MI) after the diagnosis of RA. The case and control groups were matched by sex and disease duration. RA activity and severity were determined by DAS28 scores and HAQ questionnaires, ultrasonography of synovitis. Information about traditional (BMI, smoking history, diabetes mellitus, primary arterial hypertension) and disease-specific risk factors was thoroughly gathered. AIP (atherogenic index of plasma (log10 TG/HDLC) was calculated. Brachiocephalic artery hemodynamic parameters, IMT and plaques were assessed using the high resolution B mode and Doppler-mode ultrasound.

Results: Patients with MI (myocardial infarction) were older in comparison to control RA patients, mostly females $(85 \%)$. The case and control groups were matched by disease duration and gender. One of the classic risk factors for $95 \%$ of patients with $\mathrm{MI}$ had been suffering from arterial hypertension (HTN). The odds ratio for MI patients with HTN was 12 (95\% Cl 1.5 to 95.4). Seropositivity, erosions and synovitis of small joints, joint replacement surgery was similar for case and control groups. Seropositivity was found in $95 \%$ of the case group. Despite that, RF (rheumatoid factor) was more presented in case group. High RA disease activity (DAS 28 above 5.1) was observed among $55 \%$ of patients with $\mathrm{MI}(\mathrm{p}=0.007$ ). The odds ratio for MI patients with a high disease activity (DAS $28>5.1$ ) was $3.95(95 \% \mathrm{Cl} 1.40$ to 11.13$)$. About $50 \%$ of cases were smokers. Smoking status, BMI, diabetes, atherogenix index did not differ between cases and controls. IMT (intima media thickness) $d x$ et sin were age dependent, correlated with hemodinamicaly non-significant atherosclerotic plaques. Furthermore, atherosclerotic lesions were not more pronounced in RA patients with versus without CVD. Non-hemodinamicaly significant atherosclerotic plaques and IMT sin were associated with hypertensive disease.

Conclusion: In our case-control study, MI was observed in older individuals RF positive, being positively associated with systolic arterial hypertension. Increased risk to MI was found in patients with high disease activity evaluated as DAS 28 above 5.1 .

Keywords: Intima media thickness (IMT); Atherosclerotic plaques; Myocardial infarction; Rheumatoid arthritis; Cardiovascular disease

\section{Introduction}

In the general in both USA and Western European countries [1], the risk of CVD has decreased in the past decades, while in RA the risk of CVD does not appear to have changed [2]. New evidence still portrays an association between higher cummulative inflammatory burden and increased CVD (cardiovascular disease) risk in RA [3].

$\mathrm{CV}$ (cardiovascular) morbidity is enhanced and there is an increased prevalence of all stages of atherogenesis, from endothelial dysfunction to increased intima media thickness and plaques in carotid arteries, to fatal and non -fatal myocardial infarction [4].

Patients with RA appear to be more prone to accelerated atherosclerosis in addition to plaque instability and rupture related to 
inflammation that contributes to more severe acute coronary syndromes [5]. Therefore screening for asymptomatic atherosclerotic plaques by use of carotid ultrasound may be considered as part of the CVD risk evaluation in patients with RA.

The objective of the present study was to investigate the relationship between $\mathrm{CV}$ events (MI) and atherosclerotic plaques in whole brachiocephalic branch arteries, traditional CV risk factors as well as RA (rheumatoid arthritis) disease activity in rheumatoid arthritis patients.

\section{Material and Methods}

\section{Patients}

This was a case-control study nested in a prospective cohort of patients with RA that started in 2011.

Consecutive patients who fulfilled the 2010 criteria [6] for RA, with disease duration $>6$ weeks and without prior disease-modifying antirheumatic drug use, were included in the study.

Comprehensive information on comorbidities (including CVD) and the course of the disease was gathered on a regular basis and input in an electronic database. We had availability to the medical files of each patient, including the periods before and after diagnosis of RA, so the registration of comorbidities and medical events was thorough.

The cases for this study were the patients who had developed a first MI after the diagnosis of RA or who had developed a first-time episode of an unstable angina (UA). The cases were being selected based on the fact whether the diagnosis of MI or UA was made by the cardiologist. The presence of ischaemic symptoms, ECG changes and raised cardiac enzyme levels were verified according to the patient's medical records. Cases with UA were selected in case the presence of multiple coronary vessel disease was confirmed by angiography indicating the need for a bypass surgery or a percutaneous transluminal coronary angioplasty. Controls were selected randomly from the cohort of patients with RA, with the objective to have similar disease duration (exposition time to inflammation) for cases and controls.

\section{Assessment of disease activity, severity and seropositivity}

In the cohort, disease activity was prospectively assessed using the DAS28.

The DAS28 is a measuring tool of disease activity in rheumatoid arthritis. DAS stands for 'disease activity score' and the number 28 refers to the 28 joints that are examined in this assessment DAS 28, it is calculated from the 28 -tender joint count, 28 -swollen joint count, Creactive protein and the patient's global assessment of disease related general health on a visual analogue scale. Disability was assessed using the disability index of the Health Assessment Questionnaire (HAQ).

Disease severity was assessed by using $\mathrm{X}$-ray proved erosions in small hand and feet joints, musculoskeletal sonography of synovial joints, as well as performing joint replacement surgery.

In addition, other variables were gathered including rheumatoid factor (RF) status, anti CCP status- seropositivity. The test results were divided into positive or negative according to the reference ranges and testing methods. RF testing was performed by nephelometry for mostly IgM RF, or lattex agglutionation assay for IgG RF. Anti-CCP testing was performed by enzyme immunoassay. Positive results were defined by the reported clinical laboratory standards, as follows: RF positivity was $14 \mathrm{IU} / \mathrm{ml}$, and CCP positivity was $4.0>\mathrm{U} / \mathrm{ml}$.

\section{Assessment of cardiovascular risk factors}

Traditional cardiovascular risk factors were obtained including male gender, obesity, hypertension, diabetes mellitus, smoking, dyslipidemia with high triglyceride levels, low serum High density lipoprotein (HDL) and high LDL levels. Lipids were assessed from serum samples. The atherogenic index was calculated. It is defined as the base 10 logarithm of the ratio of plasma triglyceride to high density lipoprotein cholesterol.

Body mass index (BMI) was calculated from height and weight at baseline. Diabetes mellitus was regarded to be present if the diagnosis was made before the event or censoring. Hypertension was regarded to be present if the diagnosis was made and antihypertensive medication was prescribed before the event or censoring.

Information on smoking history and occurrence of cardiovascular disease (coronary heart disease, heart failure) was assessed in entry visits and obtained from medical records or patients.

\section{Carotid ultrasound imaging}

All study participants underwent carotid ultrasonography, which was performed by an experienced research sonographer. Carotid arteries haemodynamic parameters were measured using high resolution B-mode, M-mode and Doppler-mode ultrasound. The carotid arteries were imaged with an 8.0-10 Mhz linear array ultrasound transducer GE Vivid 7 carotid ultrasound program. The standardized protocol was used to optimize and acquire images of the common, bifurcation, and internal segments of each carotid artery as well as the rest of brachiocephalic arteries. The sonographer acquired 4 standardized B- mode images and Doppler flow measurements from the right and left sides of the brachiocephalic trunk arteries. The first image was of the distal common carotid artery and the remaining 3 were centered on the site of maximum near and far wall thickening in the proximal internal carotid artery or carotid bulb. IMT measurements were obtained at each of the near and far walls of the right and left common carotid arteries and right, left internal carotid artery. The results were recorded for grading of the carotid artery intima-media thickness (IMT) and carotid plaque. The maximal IMT of each of these was obtained by averaging the maximal measurements of the near and far walls and the right and left sides. Then a composite maximal IMT was calculated by averaging the common and internal carotid measurements. Results were exposed in millimeters. In brief, the participants were examined in the supine position with slight hyperextension of the neck. Both extracranial carotid arterial systems were extensively scanned in multiple planes to optimize identification of atherosclerosis, which was defined as discrete plaque protruding into the lumen at least $50 \%$ beyond the diameter of the surrounding wall. Doppler interrogation was performed to evaluate the presence of significant ( $\geq 50 \%$ lumen diameter) reduction. Intima media thickness was measured from end-diastolic (minimum dimension) M-mode images of the far wall of the distal common carotid artery. Intimamedia thickness was not measured in a location containing plaque. Mean values of right and left intima-media thickness were presented. Reproducibility of intima-media thickness and detection of plaques has been well documented. 
Citation: Stumberga ES, Krumina G, Mikazane H, Senkane S, Ziediana L (2017) Are Brachiocephalic Atherosclerotic Plaques to be considered as Predictors of Myocardial Infarction in Rheumatoid Arthritis Patients?. J Arthritis 6: 248. doi:10.4172/2167-7921.1000248

Page 3 of 6

All ultrasound examinations were performed by a single sonographer. The sonographer was blinded to the group identity of the study subjects.

Data collection procedures: This study was approved by the Riga Stradina University Committee for Research Ethics. All subjects have submitted a written consent prior to study examinations.

\section{Statistical analysis}

The statistical analyses were carried through by using IBM SPSS statistics V.22.0 (IBM, Armonk, New York, USA). Normally distributed continuous data were expressed as mean and SD, and non-normally distributed continuous data as median and IQR. Categorical variables were presented as numbers and percentages. Cases of MI and controls were compared by disease activity variables and risk factors using the Mann-Whitney U test (for continuous variables) or the Pearson's $\mathrm{X}^{2}$ test or Fisher's exact test (for dichotomous variables).

\section{Results}

There were 92 patients (among them females 81\%) with RA at the moment of case and control selection. They ranged in age from 40 to 84 years. Twenty patients were selected as cases; they had experienced their first myocardial infarction (MI) after the diagnosis of RA. Seventy two controls were randomly selected. The case and control group were matched by disease duration and gender. Patients with MI had median disease duration of 3.5 years (IQR 0.62-10.75) ( $\mathrm{p}=0.421)$.

In our study patients with MI were mostly females $85 \%$, although gender did not differ statistically significantly between case and control groups. They were older than control group patients with a median age of $66(58.25-72.75)$ years $(\mathrm{p}=0.017)$. However the age of menarche and menopause of RA patients was comparable between case and control groups.

Out of the classic risk factors, we found out that the patients with MI had suffered from arterial hypertension (HTN) in $95 \%$ of cases, but in the control group only $61.1 \%(\mathrm{p}=0.004)$. The OR of HTN and occurrence of MI was 12 (95\% CI 1.5 to 95.4$)$

Body mass index (BMI) in all RA patients was evaluated. Mean BMI in case group was 28.18 ( \pm 5.39$)$ vs. control 26.99( \pm 4.88$),(\mathrm{p}=0.347)$. After World Health Organization produced standards which conclude that BMI of 25 to 30 should be considered overweight, we can describe our patients as overweight.

Diabetes was found in $10 \%$ of cases vs. $5.6 \%$ of control group, nonsignificantly associated with MI cases $(\mathrm{p}=0.61)$.

Mean of atherogenic index (AIP) was calculated in case group $0.14( \pm 0.29)$ vs. control 0.09 ( \pm 0.23$)$. A statistically significant association MI cases with atherogenic index was not found $(\mathrm{p}=0.405)$. We also evaluated patients with raised AIP index and found that $35 \%$ of cases had raised AIP index, but again non statistically significantly differing between case and control groups.

Despite the fact that almost half of the case group patients - about $50 \%$ were smokers for a mean of $25.70( \pm 10.27)$ years, smoking history and duration of smoking were not statistically significantly related to MI cases. Table 1 shows the characteristics of cases and controls.

\begin{tabular}{|c|c|c|c|}
\hline & MI-controls $n=72$ & Ml+cases $n=20$ & $\mathbf{p}$ \\
\hline age, yrs, median (IQR) & $58.50(53.00-68.75)$ & $66.00(58.25-72.75)$ & 0.017 \\
\hline BMI & $26.99(4.88)$ & $28.18(5.39)$ & 0.347 \\
\hline age, when rheumatoid arthritis complaints begun & $55.14(12.20)$ & $59.40(13.24)$ & 0.178 \\
\hline age when diagnosis of rheumatoid arthritis was proved & $56.81(11.85)$ & $60.65(13.74)$ & 0.219 \\
\hline duration of rheumatoid arthritis, median (IQR) & $2.00(1.00-6.00)$ & $3.50(0.62-10.75)$ & 0.421 \\
\hline smoking history, yrs, & $25.32(12.20)$ & $25.70(10.27)$ & 0.931 \\
\hline menarche, yrs, median (IQR) & $14.00(13.00-14.00)$ & $13.00(13.00-14.50)$ & 0.226 \\
\hline menopause beggining, yrs & $49.67(4.58)$ & $50.88(3.05)$ & 0.313 \\
\hline DAS28 count (CRP) baseline, median (IQR) & $3.84(2.94-5.02)$ & $5.27(3.20-5.97)$ & 0.094 \\
\hline $\mathrm{HAQ}$, median (IQR) & $1.50(0.63-2.00)$ & $1.94(1.16-2.34)$ & 0.082 \\
\hline Atherogenic index (TC/HDL) & $0.09(0.23)$ & $0.14(0.29)$ & 0.405 \\
\hline RF(IU/ml), median (IQR) & $47.25(14.10-143.50)$ & $117.65(40.45-238.60)$ & 0.049 \\
\hline Intima media thickness $(\mathrm{mm}) \sin$ & $0.92(0.18)$ & $0.96(0.19)$ & 0.404 \\
\hline Intima media thickness $(\mathrm{mm}) \mathrm{dx}$ & $0.90(0.18)$ & $0.93(0.21)$ & 0.603 \\
\hline Anti $\operatorname{ccp}(\mathrm{U} / \mathrm{ml})$, median (IQR) & $329.6(52.4-522.0)$ & 500.0. (45.0-1000.0) & 0.345 \\
\hline Female gender & $77.8 \%$ & $85.0 \%$ & 0.75 \\
\hline Atherogenix index raised (\%) & $26.4 \%$ & $35.0 \%$ & 0.58 \\
\hline
\end{tabular}


Citation: Stumberga ES, Krumina G, Mikazane H, Senkane S, Ziediana L (2017) Are Brachiocephalic Atherosclerotic Plaques to be considered as Predictors of Myocardial Infarction in Rheumatoid Arthritis Patients?. J Arthritis 6: 248. doi:10.4172/2167-7921.1000248

Page 4 of 6

\begin{tabular}{|l|l|l|l|}
\hline Seropositivity (\%) & $83.3 \%$ & $95.0 \%$ & 0.28 \\
\hline Erosions (\%) & $44.4 \%$ & $60.0 \%$ & 0.22 \\
\hline Synovitis (\%) & $95.8 \%$ & $90.0 \%$ & 0.30 \\
\hline Joint replacement surgery (\%) & $12.5 \%$ & $5.0 \%$ & 0.69 \\
\hline Atherosclerotic plaques (\%) & $65.3 \%$ & $50.0 \%$ & 0.21 \\
\hline Atherosclerotic plaques narrowing lumen more than 50\% (\%) & $10.6 \%$ & $30.0 \%$ & 0.14 \\
\hline HTN (\%) & $61.1 \%$ & $95.0 \%$ & 0.004 \\
\hline DM (\%) & $5.6 \%$ & $10.0 \%$ & 0.61 \\
\hline Ever smoker (\%) & $38.9 \%$ & $50.0 \%$ & 0.37 \\
\hline DAS 28 >4.1(\%) & $47.2 \%$ & $70.0 \%$ & 0.71 \\
\hline DAS 28>5.1(\%) & $23.6 \%$ & $55.0 \%$ & 0.007 \\
\hline $\begin{array}{l}\text { Values are mean (SD), median (p25-p75) or number (\%). HAQ: Health Assessment Questionnaire; Anti CCP: Anti-citrullinated protein antibodies; RF: Rheumatoid } \\
\text { Factor; DM: Diabetes mellitus; DAS 28: Disease activity score; HTN: Arterial hypertension; Sin: Sinistra; dx: Dexta; yrs: Years }\end{array}$ & \\
\hline
\end{tabular}

Table 1: Characteristics of patients with MI and control subjects.

Cases and controls had comparable age of beginning of RA complaints, respectively 59.40( \pm 13.24$)$ vs. $55.14( \pm 12.20),(\mathrm{p}=0.178)$.

Observing seropositivity of the patients, there were $95 \%$ of cases seropositive vs. control $83.3 \%$. The difference was not statistically significant $(p=0.280)$. We evaluated the presence of anticitrullinated protein (ACPA) antibodies and rheumatoid factor (RF). $\mathrm{RF}(\mathrm{IU} / \mathrm{ml})$ was detected in blood tests and found more present in case group, the median 117.7 (40.5-238.6) vs. control 47.3(14.1-143.5). High levels of RF values were statistically significantly related to MI cases, $(\mathrm{p}=0.049)$

$\mathrm{X}$ rays of small joints of hands and feet, revealed erosions in small joints in $60 \%$ of MI patients vs. control patients $44.4 \%$, but detectable synovitis in musculoskeletal ultrasound was found in $90 \%$ of RA cases with MI and $95.8 \%$ of control patients. In total 5\% of RA case group patients vs. control $12.5 \%$ needed total joint replacement surgery.

The mean RA disease activity evaluated as (DAS28) did not differ between case and control groups. However, when dividing different patients in groups on the basis of a moderate activity, DAS28 above 4.17 and high disease activity DAS 28 above 5.1 was observed statistically significant results in patient group with a high disease activity. There were $55 \%$ of case patients with high level of disease activity (DAS $28>5.1$ ) and only $23.6 \%$ of control group patients, ( $\mathrm{p}=0.007)$. The OR of (DAS 28>5.1) level and occurrence of MI was 3.95 (95\% CI 1.40 to 11.13 )

Health Assessment Questionnaire (HAQ) for functional status assessment was done. The HAQ score did not differ between case and control groups. The value of HAQ index was between moderate to severe in both groups [7].

Measurement of the intima-media thickness (IMT) of the far wall of the common carotid artery by high resolution ultrasonography has been established as a clinically useful index for identifying early -stage atherosclerosis. After performing neurosonological examinations no statistically significant difference in CIMT(carotid artery intima-media thickness) $d x$ et sin between cases IMT sin mean $0.96 \mathrm{~mm}( \pm 0.19)$; IMT dx mean $0.93 \mathrm{~mm}( \pm 0.21)$ and controls IMT $\sin$ mean $0.92 \mathrm{~mm}( \pm$
0.18 ; IMT $\mathrm{dx}$ mean $0.90 \mathrm{~mm}( \pm 0.18)$ could be found respectively CIMT $\sin (\mathrm{p}=0.404)$ vs. CIMT $\mathrm{dx}(\mathrm{p}=0.603)$. Nevertheless it should be noted that in the latest ESH/ESC hypertension guidelines (2013) carotid IMT $>0.9 \mathrm{~mm}$ has been re-confirmed as a marker of asymptomatic organ damage $[8,9]$.

Duration of RA, disease activity (DAS28), synovitis, erosions, smoking history, gender, seropositivity, the age of menarche and menopause for female patients, as well as AIP did not have any relationship with CIMT $(\mathrm{p}>0.05)$.

IMT $\mathrm{dx}$ had statistically significant correlation with IMT $\sin$ $(\mathrm{r}=0.82, \mathrm{p}>0.0001)$ as well as the age of patients $(\mathrm{r}=0.26, \mathrm{p}=0.011)$, the same about $\operatorname{IMT} \sin (\mathrm{r}=0.22, \mathrm{p}=0.033)$.

CIMT sin of the RA patients had correlation with one more traditional risk factor as hypertension IMT $\sin \mathrm{t}(90)=-2,137 ;(\mathrm{p}=0.035)$. For the patients who had HTN the mean of IMT sin was $0.96( \pm 0.19)$ vs. patients without hypertensive disease IMT sin mean $0.87( \pm 0.17)$.

IMT of our patients had correlation with atherosclerotic lesions of brachiocephalic vessels without hemodynamically relevant stenoses, respectively IMT $\sin (\mathrm{p}=0.004)$, IMT $\mathrm{dx}(\mathrm{p}=0.016)$. However there was no correlation found CIMT with atherosclerotic plaques of brachiocephalic vessels narrowing lumen more than $50 \%$.

Furthermore, atherosclerotic lesions were not more present in RA patients with versus without CVD. Atherosclerotic plaques in brachiocephalic vessels resulting in $<50 \%$ luminal stenosis were found in $50 \%$ of the cases; $65.3 \%$ in the control group $(\mathrm{p}=0.213)$, but plaques causing $>50 \%$ lumen had $30 \%$ of CVD patients and $10.6 \%$ - in the control group $(\mathrm{p}=0.137)$ we divided patients with atherosclerotic plaques below $50 \%$ lumen narrowing and $\geq 50 \%$ of lumen narrowing. A narrowing of $50 \%$ of the lumen diameter is considered to be hemodynamically significant.

Atherosclerotic plaques (hemodynamically significant and nonsignificant) were not age and gender dependent ( $p>0.05)$, did not have correlation with age of RA initial presentation. 
Disease duration, disease activity and atherosclerotic plaques. Different patient groups were divided on the basis of hemodynamically significant and non-significant stenosis, graded above and below $50 \%$ lumen narrowing did not differ significantly from each other according to disease duration as well as disease activity.

Neither disease activity (low, moderate or high evaluated as DAS 28 score), nor HAQ-the disability index, seropositivity, erosive findings in small joints had some relationship with atherosclerotic plaques $(\mathrm{p}>0.05)$.

Well known risk factors and atherosclerosis. The development of atherosclerotic plaque is related to the exposure to recognized cardiovascular studied risk factors. The commonly recognized risk factors include elevated cholesterol levels, cigarette smoking, diabetes and hypertension.

From traditional risk factors non-stenosing atherosclerotic plaques were related to hypertensive disease $(\mathrm{p}=0.022)$. Smoking history, diabetes, Atherogenix index were not related to atherosclerotic changes in brachiocephalic vessels regardless of the plaque size and lumen narrowing.

\section{Discussion}

Patients with established rheumatoid arthritis (RA) are at increased risk of subclinical and clinical cardiovascular disease [10].

There is an increased presence of cardiovascular morbidity in both men and women [11].

In line with EULAR 2016 recommendations screening for asymptomatic atherosclerotic plaques by using carotid ultrasound may be considered a part of the CVD risk evaluation in patients with RA [5].

The goal of this study was to compare the extent of carotid atherosclerosis between patients with MI and control group subjects assessing atherosclerotic changes in all brachiocephalic vessel branch arteries. Therefore, a high resolution B-mode ultrasound measurement of carotid artery IMT was done. Intima-media thickness (IMT) is a marker of subclinical atherosclerosis, however raised IMT can be related to a number of classic vascular risk factors, such as hypertension, smoking and cholesterol, homocysteine levels, Creactive protein and the presence of a metabolic syndrome or coronary artery disease [12].

In our study CIMT sin was associated with HTN, IMT $d x$ et sin was age dependent and correlated with hemodinamicaly non-significant atherosclerotic plaques of brachiocephalic vessels. However IMT did not differ between MI cases vs. controls.

RA disease duration and disease activity have revealed to be related to plaque size and vulnerability in patients with RA $[13,14]$.

According to our study results, neither disease duration nor activity assessed as DAS 28 score (low, moderate or high) had some relationship with atherosclerotic plaques regardless of their size, lumen reduction diameter $(\mathrm{p}>0.05)$. However plaque vulnerability mainly diagnosed by $\mathrm{B}$ mode ultrasound is currently not satisfactorily accessible by ultrasound methods therefore was not assessed in our study [15].

Dessein et al. have reported that the presence of atherosclerotic plaques is related to patient age, sex and CV risk factors such as smoking and blood pressure [16].
According to our data, atherosclerotic plaques (hemodynamically significant and non-significant) were not age and gender dependent, only atherosclerotic plaques (hemodynamically non-significant) were related to hypertensive disease.

Traditional risk factors such as age, hypertension are related to an increased cardiovascular risk in RA $[17,18]$.

It is in line with our study findings, where patients with MI were associated with hypertensive disease and were older by age than control group patients. Pathopysiological data have delivered support for the notion of disease duration as a risk factor for CVD as it has been reported that accelerated atherosclerosis appears to be more severe in RA patients with established disease [18].

Therefore first of all we gathered patients with comparable disease duration and secondly assessed the distribution of atherosclerotic plaques (with hemodinamically relevant stenosis and without) and disease duration. The results of our study did not show statistically different results in terms of relationship with grade of stenosis and disease duration.

In reference to the EULAR recommendations that indicate a disease duration $>10$ years as a risk factor for CVD, we were able to identify patients with coronary events even at an earlier period of RA disease. Our study results, where we could detect higher prevalence of CVD during the first 10 years of RA, are in line with findings from several other studies that suggest the excess risk of CVD is already present in the earlier stage of RA [2].

Another disease-related factor that may mediate atherosclerosis is the presence of autoimmunity or immune dysregulation. RF is significant predictors of $\mathrm{CV}$ events in both those with and those without rheumatic diseases [19].

It is believed that patients with RA who are seropositive for rheumatoid factor have a higher risk of $\mathrm{CV}$ events than seronegative patients with RA [20].

In study of Liang et al. [19] was found that a positive RF test was a significant predictor of MI and death [19].

In our study patients RF positive were associated with MI. Fortunately none of our patients had letal outcome, but tendency that patients with $\mathrm{RF}$ are more prone to $\mathrm{CVD}$, respectively to MI, was confirmed in our study.

Different patient groups divided based on disease activity did not differ significantly from each other in terms of association with $\mathrm{CV}$ events. However, when looking at the differences, patients with high level of disease activity appear to be at a substantially higher risk of developing CVD (MI) in comparison to the patients with lower disease activity levels.

The results of our study suggest that increased disease activity calculated as (DAS28>5.1) increased CV events (MI), despite the fact that erosions of small joints, synovitis detected by ultrasound, HAQ questionnaire results did not appear to be significantly related to $\mathrm{CV}$ events. Additional research is necessary in order to examine further the relationship between the individual RA disease activity, severity components and $\mathrm{CV}$ events.

\section{Conclusion}

Our study results show that inflammation and RF positivity in RA may contribute more specifically to more severe acute coronary 
Citation: Stumberga ES, Krumina G, Mikazane H, Senkane S, Ziediana L (2017) Are Brachiocephalic Atherosclerotic Plaques to be considered as Predictors of Myocardial Infarction in Rheumatoid Arthritis Patients?. J Arthritis 6: 248. doi:10.4172/2167-7921.1000248

Page 6 of 6

syndromes that may be more seriously related to the presence and severity of high disease activity expressed as DAS 28 above 5.1 than with disease duration. Among traditional risk factors, the patient age and hypertensive disease are the ones most strongly linked to CV (MI) events.

\section{Acknowledgment}

The author acknowledges the assistance given by consultants Ieva Salmane at the Riga Stradin's University.

\section{References}

1. Gabriel SE (2001) The epidemiology of rheumatoid arthritis. Rheum Dis Clin North Am 27: 269-281.

2. Gabriel SE, Crowson CS, Kremers HM, Doran MF, Turesson C, et al. (2003) Survival in rheumatoid arthritis: a population -based analysis of trends over 40 years. Arthritis Rheum 48: 54-58.

3. Myasoedova E, Chandran A, Ilhan B, Major BT, Michet CJ, et al. (2016) The role of rheumatoid arthritis (RA) flare and cumulative burden of RA severity in the risk of cardiovascular disease. The role of rheumatoid arthritis flare and cumulative burden of RA severity in the risk of cardiovascular disease. Ann Rheum Dis 75: 560-565.

4. Peters MJL, Symmons DPM, McCarey D, Dijkmans BAC, Nicola P, et al. (2010) EULAR evidence-based recommendations for cardiovascular risk management in patients with rheumatoid arthritis and other forms of inflammatory arthritis. Ann Rheum Dis 69: 325-331.

5. Agca R, Heslinga SC, Rollefstad S, Heslinga M, McInnes IB, et al. (2017) EULAR recommendations for cardiovascular disease risk management in patients with rheumatoid arthritis and other forms of inflammatory joint disorders: 2015/2016 update. Ann Rheum Dis 76: 17-28.

6. Aletaha D, Neogi T, Silman AJ, Funovits J, Felson DT, et al. (2010) 2010 Rheumatoid arthritis classification criteria: an American College of Rheumatology/European League Against Rheumatism collaborative initiative. Ann Rheum Dis 69: 1580-1588.

7. Felson DT, Anderson JJ, Boers M, Bombardier C, Chernoff M, et al. (1993) The American College of Rheumatology preliminary core set of disease activity measures for rheumatoid arthritis clinical trials. Arthritis Rheum 36: 729-740.

8. O'Leary DH, Polak JF, Kronmal RA, Manolio TA, Burke GL, et al. (1999) Carotid-artery intima and media thickness as a risk factor for myocardial infarction and stroke in older adults. N Engl J Med 340: 14-22.
9. Zanchetti A, Hennig M, Hollweck R, Bond G, Tang R, et al. (2009) Baseline values but not treatment-induced changes in carotid intimamedia thickness predict incident cardiovascular events in treated hypertensive patients: findings in the European Lacidipine Study on Atherosclerosis (ELSA). Circulation 120: 1084-1090.

10. Holmquist M, Gränsmark Ängla Mantel E, Alfredsson L, Lennart TH, Wallberg-Jonsson S (2013) Occurence and realtive risk of stroke in incident and prevalent contemporary rheumatoid arthritis. Ann Rheum Dis 72: 541-546.

11. Solomon DH, Goodson NJ, Katz JN, Weinblatt ME, Avorn J, et al. (2006) Patterns of cardiovascular risk in rheumatoid arthritis. Ann Rheum Dis 65: 1608-1612.

12. Simova I (2015) Intima-media thickness: appropriate evaluation and proper measurement, described. E-Journal of Cardiology Practice.

13. Semb AG, Rollefstad S, Provan SA, Kvien TK, Stranden E, et al. (2013) Carotid Plaque characteristics and disease activity in rheumatoid arthritis. J Rheumatol 40: 359-368.

14. Wallberg-Jonnson S, Ohman M, Rantapaa-Dahlqvist S (2004) Which factors are related to the presence of atherosclerosis in rheumatoid arthritis? Scand J Rheumatol 33: 373-379.

15. Valdueza JM, Schreiber SJ, Roehl JE, Klingebiel R (2017) Neurosonology and Neuroimaging of Stroke.

16. Dessein PH, Norton GR, Woodiwiss AJ, Joffe BI, Wolfe F (2007) Influence of nonclassical cardiovascular risk factors on the accuracy of predicting subclinical atherosclerosis in rheumatoid arthritis. J Rheumatol 34: 943-951.

17. Radovits BJ, Popa-Diaconu DA, Popa C, Eijsbouts A, Laan RF, et al. (2009) Disease activity as a risk factor for myocardial infarction in rheumatoid arthritis. Ann Rheum Dis 68: 1271-1276.

18. Arts Elke EA, den Broeder Alfons A, Popa Calin D, Fransen J, Piet LCM, et al. (2015) The effect of disease duration and disease activity on the risk of cardiovascular disease in rheumatoid arthritis patient. Ann Rheum Dis 74: 998-1003.

19. Liang KP, Kremers HM, Crowson CS, Snyder MR, Therneau TM, et al. (2009) Autoantibodies and the risk of cardiovascular events. J Rheumatol 36: $2462-2469$

20. Goodson NJ, Wiles NJ, Lunt M, Barrett EM, Silman AJ, et al. (2002) Mortality in early inflammatory polyarthritis: cardiovascular mortality is increased in seropositive patients. Arthritis Rheum 46: 2010-2019. 